\section{EUROPEAN \\ POLYGRAPH}

PUBLISHED SEMI-ANNUALLY

2021 VOLUME 15 NUMBER 2 (54)

DOI: 10.2478/EP-2021-0005

\title{
From a Rigid Cover to an Elastic One Via a Blanket too Short. An Adaptive Polygraph Approach'
}

\author{
Avital Ginton \\ $\mathrm{PhD}$; \\ Independent practitioner and researcher, AV-GN, Polygraph, Applications \& Research, and part time lecturer, \\ Faculty of Law, Tel-Aviv University (C/O Prof. Shlomo Shoam), Israel.; ginton@inter.net.il
}

Key words: Adaptive Polygraphy, Relevant Issue Gravity, RIG, Comparison Question Test, CQT, Polygraph, Deception Detection

\begin{abstract}
The Polygraph test or the Psychophysiological Detection of Deception is a short blanket that cannot cover everything without paying in errors; a clever polygraph examiner and a wise usage of polygraph must make a choice whether to cover the feet or the head with this short blanket and conduct the examination accordingly. But a wiser approach should look to turn the short blanket into an elastic cover that can deal differently with different people and different situations.

Following two-three decades of blessed efforts to develop strict standardization in the field, the time has come to start steering the polygraph ship back to greater flexibility and creativity,

\footnotetext{
${ }^{1}$ Based on an oral presentation in the $48^{\text {th }}$ annual seminar of the American Polygraph Association, 2013, Orlando, FL. USA.

Sciend ${ }^{\odot}$ year of first publication Author(s). This is an open access article distributed under the Creative Commons Attribution-NonCommercial-NoDerivs license http://creativecommons.Org/licenses/by-nc-nd/3.0/
} 
this time relying on scientific thinking and knowledge. Thus, contrary to the existing trend in the field, I call to drive modern Polygraphy towards developing a scientifically based approach that follows the motto of "Different Things to Different People and Different Situations". In other words, I call for developing an adaptive approach or Adaptive Polygraphy. The Relevant Issue Gravity (RIG) Theory (Ginton, 2009) is presented here as a major theoretical and practical carrier for evolving and shaping the Adaptive Polygraphy. The article analyzes the current situation and draws some lines to follow in developing an Adaptive Polygraphy approach.

\section{Preface}

Among the most Frequently Asked Questions (FAQ) regarding polygraph testing, we can find the question of "How accurate is the Polygraph?" or the more sophisticated version of it "What do we have more, False-Positive or False-Negative errors and what are their respective rates?". Beyond the fact that there are a variety of techniques and usages, which might affect the accuracy, I would like to point out the existence of three distinct approaches or attitudes to these questions that are based on different assumptions, which might result in diverse answers to those questions as well as different implications. I would term these three generic approaches: A Rigid Cover, A Blanket Too Short, and An Elastic Cover.

\section{A Rigid Cover - Assumptions:}

1. Accuracy rate of Polygraph tests is an actual figure representing the real quality of the test and not just a statistical manipulation. Our task is to find proper ways to expose this existing figure (or figures, in case we differentiate between various techniques or formats of tests).

2. A certain percentage of the test's outcomes is not clear enough to make a call and deems Inconclusive.

3. Accuracy of detection and rate of Inc outcomes might be different for Deceptive and Truth-teller examinees. Our task is to expose these existing differences.

A "Competition" between several fixed numbers, the results of independent studies, ended up in some sort of averaging them, with the highest methodological achievement of using Meta-Analysis as a means for estimating the final figure (be it $90 \%, 85 \%, 70 \%$, etc.).

I call this approach A Rigid Cover because it ends up with certain fixed figures claimed to be the estimated accuracy rates of the test, as genuine characteristics 
of it, which indicate the proportion of correct outcomes (True Positive and True Negative) covered by the test. Almost all of the tens validity research cited in the APA report of the Meta-analytic Survey of Validated Polygraph Techniques (APA, 2011) are products of this approach. There might be disagreements about the size of the figures, but the debate is about which figure represents better the genuine accuracy of the test. Thus, it is based on the assumption that there is such a thing as "a genuine accuracy characteristic of the test".

\section{A Blanket Too Short - Assumptions:}

1. Both types of errors are inherently embedded in the tests, and the detection rates cannot reach perfection.

2. The actual figures of the detection and inconclusive rates are subject to our manipulations in conducting the tests or analyzing the outcomes. It is mostly a tradeoff manipulation that changes the Inconclusive and error rates (FP vs FN).

3. We are acting within a pay-off matrix in which it is possible to increase one sort of detection and accuracy rate at the expense of lowering the other.

4. The philosophy or policy held by the examiners or their organizations with regards to the preferred cost-benefit relationship that is manifested in the pay-off matrix affects these rates.

I call it "A Blanket Too Short" - having in mind a person who has to decide whether to pull the short blanket to cover his head and shoulders and by that exposing his feet to the cold, or to cover his feet and leave his shoulders and head to the cold. The size of the blanket is fixed, but the way it affects the person who uses it is very much under his control. An example of that will be the use of different cut-scores in making a decision about the veracity of the examinee (e.g., Elaad, 1999; Ginton, 2013; Honts and Driscoll, 1987; Krapohl, 2005).

\section{An Elastic Cover - Assumptions:}

A third approach involves an additional assumption that precedes the four that stand behind the "blanket too short" viewpoint, as follows:

1. It is possible to act at the level of an individual exam to increase the overall detection or accuracy rate while keeping the probability of automatically paying in errors at a low level. 
2. Both types of errors are inherently embedded in the tests, and the detection rates cannot reach perfection.

3. The actual detection and inconclusive rates are subject to our manipulations in conducting the tests or analyzing the outcomes. It is mostly a trade-off manipulation that changes the Inconclusive and error rates (FP vs. FN).

4. We are acting within a pay-off matrix in which it is possible to increase one sort of detection and accuracy rate at the expense of lowering the other.

5. The philosophy or policy held by the examiners or their organizations with regards to the preferred cost-benefit relationship that is manifested in the pay-off matrix affects these rates.

I call it "Elastic Cover" since this approach aims to optimize the way the examination is conducted and analyzed per individual case, resulting in elasticity in these regards.

Two things should be asked concerning "The Elastic cover": Is it really possible to affect the results without introducing uncontrolled, chaotic consequences? And is it the right thing to do in pursuing the truth?

Whereas to the first question, many examiners believe that their behavior can push the results towards each of the two possible outcomes - DI or NDI, by manipulating the pretest interview without increasing the irrelevant noise, no research was published yet that demonstrates it. Nonetheless, I take the liberty to mention a small experiment conducted in 2004 as part of an $R \& D$ project in Israel that never got finished ${ }^{2}$.

It was a mock crime simulating smuggling drugs and cold weapons into an "Airport".

Four eight-people groups were recruited from the community. Two were "smugglers," and the other two, non-smugglers. After entering the 'airport', an 'incriminating information' cast suspicion on them of smuggling and concealing a weapon and drugs in a hidden place in the airport halls, and they had to go through a polygraph test. A matrix of incentives and punishments were applied to induce motivation. The experiment was conducted under IRB conduct to ensure the safety of the subjects. There was an even number of guilty and innocent subjects.

\footnotetext{
2 The project was aimed to develop a non-intrusive unnoticed device for psychophysiological deception detection. The referred experiment which has never been sent for publication was conducted by Ginton Avital and Kleiner Murray.
} 
The subject passed CQT followed by two CIT tests. Half of them went through a standard pretest interview, and the pretest of the other half included a special intensive comparison questions' stimulation. As expected, results indicated that those who went through an intensive stimulation of the comparison sphere significantly moved towards the NDI side. Thus, it demonstrated empirically that the examiner's behavior could maneuver the outcomes. Still, an important question was left on a different level: Suppose it is found that these maneuvers improve correct results. Should these controlled manipulations be used, and when?

Turning a 'Blanket Too Short' into an 'Elastic Cover' that adapts itself to fit the actual examination at hand by adjusting the pretest and the test to the specific examinee and the particular circumstances rather than applying a stiff standardized technique needs a profound understanding of the factors involved in polygraph testing, followed by analyzing their relative loads in each individual examination or subgroups of them.

In principle, these tailor-made examinations can bring, so to speak, the feet and shoulders or head under the cover by optimally reaching and exploiting the testing potential embedded in the polygraph and its methods (Ginton, 2013).

It should be stressed that the transition from a factory-made polygraph test mode to a tailor-made polygraph mode does not mean to play against the standardized "one size fits all" by introducing more formats, allowing each examiner to choose his/her preferable format for a particular case, be it the $\mathrm{X}$ format or rather the more suitable $\mathrm{Y}$ format. The customized or tailored approach that this article offers goes far beyond that, as will be presented throughout the article.

\section{"From Rigid to Elastic Cover via a Blanket too short" is not only a metaphor; it is also a call for scientifically based Adaptive Polygraphy because the elastic cover is where the future of the polygraph lies.}

\section{A Bit of Background}

Since the early works of Keeler in the 30ths (Keeler, 1933), Reid in the 40ths (Reid, 1947), Backster in the 60ths (Backster,1963), and other ancestors of modern Polygraphy in the $20^{\text {th }}$ century, we have witnessed introductions of a variety of polygraph techniques, methods and tactics, (for historical perspective see Krapohl \& Shaw, 2015; Matte, 1996; NRC,2003; Raskin, Honts \& Kircher, 2014). Some of them included theoretical claims, while others lacked any clear spoken theoretical 
reasoning to support their specific method or justify the suggested technique variation. Nevertheless, one may assume that all of them always contained some kind of presumed rationale or justification, including the unarguable face validity statement (sometimes unspoken) "it works for me all right". Assuming examiners want to succeed in their work, if they encountered feedback that tells them they were doing very poor work, they would probably incorporate this feedback and make some changes in the way they were functioning. In the same vein, if they stick to their technique, one may assume that it really works for them. Alas, professionally, this is not enough. If we want to adopt some scientific claims or values in our profession, "It works for me" is but the very first step in the path that establishes scientific quality in the polygraph profession.

The essence of science is to move from a subjective point of view to an objective one. The method or the technique should work for every qualified person, and as long as this could not be established and proved, we are not dealing with scientific-based methods; rather, this is an art skill in the best case and a "mambo-jumbo" business in many others.

Several important steps have been taken in the last few years, mainly by the American Polygraph Association or under its umbrella, to make the polygraph a more scientific-based profession. To name a few: Validation of techniques; Models of Best Practices; Models of TDA; ASTM standards.

The leading common theme in all these pieces of work is that we need to establish research-supported rules to guide our practice and introduce standardization to the examinations, which is a fundamental brick in the psychometrics testing theory.

Due to the complexity and the multi-factorial issues dealt with by the behavioral and biological sciences, it is customary to use research methods that target the central tendencies of phenomena which are formalized in general principles and rules that concern most of the existing variance while sometimes treating the individual differences or the variation between existing situations as irrelevant noise.

When it comes to applications, some standards are developed and implemented to ensure that the applications are conducted within the framework posed by those rules. Practically, this is a must for avoiding chaos. However, because the standards are based on central tendencies, they are inefficient or even harmful to people or situations that are off these centers. 
An extreme strive for rigid standardization in the name of science, unfortunately, tends to ignore the complexity of the field; and it seems to be based on a simplistic and limited concept of what science is, let alone that there is more than just science in practicing Polygraphy.

Along with the scientific foundations of the Psychophysiological detection of deception, we should remember that much art is also involved. We should adopt the scientific methods not only in favor of standardizing the test's stages, question formats, and data analysis, but also to improve our understanding of the "art" quality found in our work rather than suppress it in the name of science and standardization.

Over standardization, in its extreme form, adversely affects creativity, open-mindedness, flexibility, and humane touch, which are very important for further developments in our area, including pursuing the significance of personal and situational differences to the understanding and practicing Polygraph testing.

We should not, in the name of science, throw away the tailor-made approach in conducting polygraph examinations that for years has characterized the work of the best polygraph examiners and shift into the standardized "scientific" mediocre kind of work.

Within a broader and more sophisticated approach, those important and necessary moves in the last few years are only the first steps, and probably, I dare to say, the easiest ones. The following steps must deal with the individuals and specific situational variance not as noise but as part of the phenomenon that needs to be systematically addressed and explained.

An example of that can be found nowadays in medicine. A clear trend to shift from the simple standardization of diagnoses and treatments to individualized or personalized medicine is taking place. It is based on pursuing individual differences between the patients in biological, psychological, and environmental aspects and applies tailor-made diagnostic yardsticks and treatments compatible with the specific variations found in that specific patient at the time.

This medical philosophy and practice, which is highly affected by the new developments in the field of the human genome, says that modern medicine should be Personalized Medicine, meaning “Different Things to Different People."

\section{Adaptive Polygraphy - Different Things to Different People and Different Sit- uations.}


Polygraph testing, or the Psychophysiological Detection of Deception, is a short blanket that cannot cover everything without paying in errors; a clever polygraph examiner and a wise usage of polygraph must choose whether to cover the feet or the head with this short blanket and conduct the examination accordingly. But a wiser approach should look to turn the short blanket into an elastic cover that can deal differently with different people and different situations.

Contrary to the existing trend in the field that adores the strict standardization, I call to start steering the ship of modern Polygraphy towards developing a scientifically-based approach that follows the motto of understanding and conducting "Different Things to Different People and Different Situations". In other words, I call for developing an adaptive approach or Adaptive Polygraphy.

That might be the only way to improve our performance beyond the glass ceiling of $85-90 \%$ accuracy and $10-20 \%$ INC rate.

Science cannot contradict nature whether we mean biological, psychological, or social life, and in philosophy - the mother of all sciences - we can find the phrase of Aristotle "The worst form of inequality is to try and make unequal things equal". Rephrased two thousand years later by Thomas Jefferson to: "There is nothing more unequal than the equal treatment of unequal people."

Probably they meant to endorse ethics and social justice, but it is also valid to other spheres, including polygraph testing. Whenever we deal with individuals and particular cases, we encounter assortments of people and circumstantial differences to be dealt with. We should not ignore the differences but rather explore ways to treat them by an adaptive approach. As a matter of fact, to a certain degree, this has been done for years. One of the very basic instructions to a polygraph examiner is to adjust the language level to the examinee and, if possible, phrase the questions using words that the examinee has used during the pretest interview. However, the Adaptive Polygraphy approach means a broader and deeper range of adjustments.

We should adapt the examination to the subjects rather than squeeze the subjects and the case to fit a standard and a rigorous test. As already mentioned above, standardization is usually built on central tendencies and a limited variety of circumstances. It seems to works all right with a generic test conducted on a generic examinee by a generic examiner and also obeys an extrapolation of the Pareto principle, which states that for many outcomes, roughly $80 \%$ of consequences come from $20 \%$ of the causes, or for many phenomena, $80 \%$ of the result comes from 
$20 \%$ of the effort. Thus, for the general society, it is beneficial to rely on central tendencies, but what about the ignored 20\%? Dealing with this is not a simple matter. A claim might be heard that in chasing this $20 \%$, we might lose a significant portion of the $80 \%$, and we should better stick to the standardized material that yields decisions in about $80-90 \%$ of the tests, $90 \%$ of them correct. This means that $76 \%$ of the total outcomes are correct decisions, 15\% INC, and 9\% errors (e.g., American Polygraph Association, 2011). In opening the field to methods and ideas that have not yet been verified, we risk worsening the situation, not to mention moving away from scientific norms.

Nevertheless, in principle, one cannot deny that if we manage to apply the "elastic cover", the situation will be improved. Unfortunately, there is still a 'small question' to be solved. How do we get there? The presented approach suggests a specific vehicle to get there - the Ginton's RIG theoretical framework (Ginton, 2009; Ginton, 2019) as put forward in the present article.

\section{The RIG theoretical framework - the carrier of the Adaptive Polygraphy}

To begin with, a few preliminary questions must be answered first. In answering these questions, we have to turn to the very basic polygraph matter and advance from there step by step.

\section{Why and What triggers the Autonomic Nervous System (ANS) reactions?}

The function of the ANS is to increase the prospects of survival. This is done by keeping internal Homeostasis and reacting to current or anticipated significant changes in the external world. Facing such significant changes results in involuntary reactions of the ANS, aimed to adjust to the changes and improve the chances to survive. The intensity of the reactions is positively correlated with how significant is the stimuli to the organism.

\section{Why do people react with Autonomic Nervous System activity changes to Psy- chological stimuli?}

Other than pure physiological functions, attaching significance to stimuli is a psychological process, and most occurred or expected changes in the environment gain their significance from psychological functions and processes such as perception, memory, learning, feeling, etc. 
Two kinds of processes are involved in attaching significance to stimuli, Bottom-Up and Top-Down. While Bottom-Up processes are mainly affected by the physical qualities of the stimuli, the Top-Down processes are driven by the individual state of mind and the psychological qualities of the stimuli.

\section{Why do people react with Autonomic Nervous system Changes when they Lie?}

The default in communication between people is transmitting the truth. Any act of communication that deviates from the default is a change that needs to be addressed by adjusting the ANS activity, i.e., physiological reaction. Thus, in general, lying is a significant event that affects/changes both parties' minds. Lying puts the liar in a risky situation due to possible adverse rebound from the surroundings. All of the above are relevant to survival. Note, however, that telling the truth might also be risky sometimes and certainly, significant on many occasions.

Given the above answers, it seems reasonable to apply a psychophysiological test to detect lying. The first technique, known as the Relevant/Irrelevant (R/IR) test, compares the physiological reactions to Relevant questions concerning the investigated suspicion to the responses measured when nonrelevant neutral questions were posed to the subject. It turned out that almost all liars react stronger to the $\mathrm{R}$ questions, but also, a significant number of the truth-tellers did so. There was a fundamental need to develop a questioning technique that pulls the truthful subjects from the group that their test points to deception.

The technique that provides this feature is the Comparison Question Test, previously known as the Control Question test - the CQT. This technique is attributed to John Reid ${ }^{3}$ in the 40s of the previous century (Raskin and Hont, 2002; Reid, 1947). One can speculate that at first, the way Reid looked at the Control questions was more a means to control for the existence of non-deception elements that stimulate the ANS response and not as a means to pull the truthful people out of the 'reacting-as-liar' group. Therefore, he used the term Control Question and not something like Extrication, Rescue, or Disengagement Questions ${ }^{4}$.

However, the CQT method manages to do more than just control for non-deception elements that stimulate the ANS response. It manages to cause a reversed differential strength of reactions between Relevant and Comparison questions in

\footnotetext{
${ }^{3}$ A different version was attributed also to Rev. Summers in the late 30s (Krapohl \& Shaw, 2015)

${ }^{4}$ Control in the Reid technique is different from its meaning in research methodology, which caused a continuous misunderstanding between polygraph practitioners and academicians.
} 
Deceptive vs. Truthful subject. Deceptive subjects react to the Relevant questions with stronger physiological responses relative to their responses to the Comparison questions, whereas the Truthful subjects react to the Comparison questions with stronger responses than their reactions to the Relevant questions. How does it happen?

In 2009 Ginton published a new concept that he termed the Relevant Issue Gravity (RIG) relating to this question. As already been mentioned (Ginton, 2013), the RIG can function as a vehicle to reach Adaptive Polygraphy. To understand it needs to go back to some essence of the RIG theoretical framework.

The Relevant Issue Gravity theoretical framework distinguishes between two fundamental elements of the CQT. The first deals with explaining the origin of the physiological responses accompanying the act of lying. The second concentrates on explaining the phenomenon of a reversed pattern of relative reactions' strength to Relevant and Comparison Questions in deceptive vs. truthful subjects. The following account focuses on the second element. Some parts literally repeated similar accounts written by the author in a previous publication (Ginton, 2019).

Upon arrival to the test, and even before that, both the Guilty and the Innocent are busy consciously and pre-consciously in cognitive and emotional mental activity related to the Relevant Issue. It is frightening for both of them, and they are very much under its influence in a way that entraps their attention. This mental and emotional preoccupation with the forthcoming examination regarding the relevant issues involves much more than just the fear of the test's possible consequences. It also contains memories, images, a stream of associations, elevated motivations, etc.

The higher the intensity of this ongoing preoccupation of the mind (cognitively $\&$ emotionally) with the Relevant Issue, the more compelling the attention invested in it, which in turn increases the preoccupation of the mind in a positive feedback loop. The more you think about it, the more your attention is stuck in; the more your attention is stuck in, the more you think about it.

It is a trap for attention resulting from what Ginton has termed: The Relevant Issue Gravity (RIG).

The Relevant Issue Gravity (RIG) is a psychological force induced by the aggregation of qualities that the relevant issue possesses, which attracts and binds the examinee's attention to it. It is the product of some general qualities that the relevant issue always possesses due to the very fact of being a relevant issue on the test, 
plus more specific, case-related characteristics, interacting with circumstantial and personal factors.

The relevant issue attracts and binds the attention of any normal examinee, whether deceptive or not, and as a byproduct causes considerable neglect of other issues or stimuli. The more significant the issue to the examinee, the greater the amount of attention he invests in it, which means an increased RIG strength, resulting in less free-floating attention available to him/her.

The RIG strength indicates the degree to which the suspect's attention is attracted to and stuck in the relevant issues, and it is a product of many circumstantial and personal factors.

The RIG can take various levels of strength, and there are good reasons to assume that, on average, the RIG strength for the deceptive subjects is stronger than for the truthful ones. (see Ginton, 2019 for more details), as illustrated in Fig. 1.

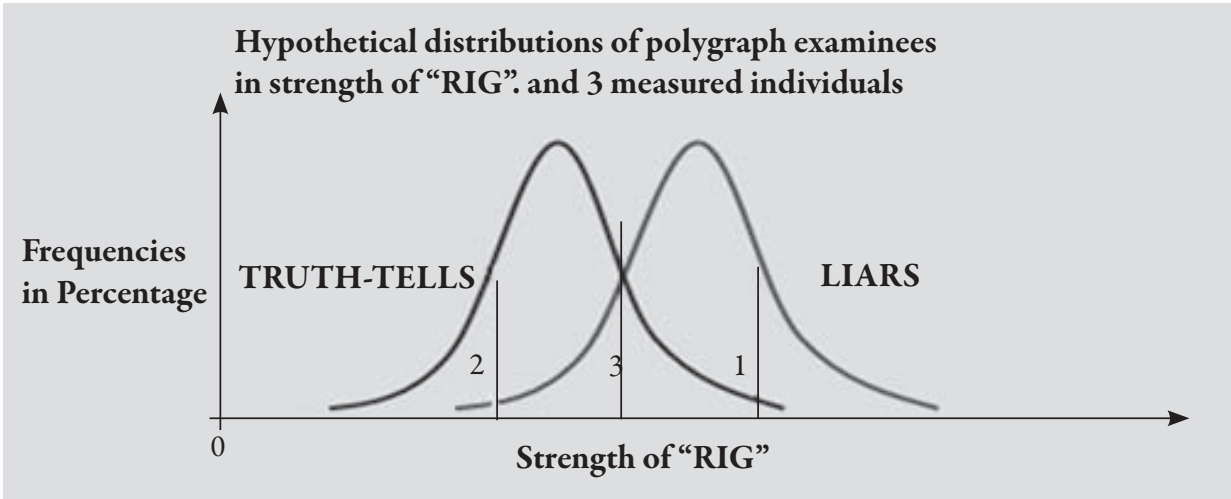

Fig. 1. Hypothetical distributions of strength of "Relevant Issue's Gravity" ("RIG") in Truth-tellers and Liars, with values of 3 individuals. In is assumed that the RIG's strength is higher for the population of liars and roughly speaking there is $90 \%$ chances that \#1 is a Liar and \#2 is a Truth-tellers while \#3 has equal chances to belong to either one the populations

In order to pay attention to the comparison question, one should first detach himself to a certain degree from the relevant sphere.

One way to measure the strength of the RIG for a particular suspect is to find how much it takes to distract the examinee's attention away from the relevant issue. The harder it is, the stronger the RIG that the examinee holds. 
This shift or change in the focus can be achieved by introducing baits to attract the examinee's attention away from the relevant sphere.

In principle, the baits can take various forms with different levels of attraction. Within the set of polygraph examinations, the examiner introduced the baits in the form of what is known to be the comparison questions and the pretest interview that leads to their formulation. Since the RIG strength for deceptive subjects is high, it is hard to detach their attention from the relevant issue sphere and shift it to the comparison one. At the same time, it is much easier to succeed in this with truthful examinees whose RIG strength is weaker.

The most important task the polygraph examiner has in the CQT is managing the diversion of the truthful examinee's attention from the relevant sphere to the comparison ones with minimum effect on the deceptive examinees. A matter which is impossible to standardize without giving room to the existing variability among cases.

The higher the success of these baits to attract the attention, the stronger will be the impact of the comparison questions and the psychophysiological reactions to them.

According to the RIG strength theory, stronger reactions to the comparison questions indicates a lower level of RIG strength and, therefore, a higher probability that the examinee belongs to the truth-tellers distribution, i.e., he/she is probably a truthful subject and vice versa.

Note, however, that if the baits are too big/strong, they might attract almost any person's attention and shift it to the comparison sphere in almost any circumstances. The opposite holds for too small or too weak baits that might fail to attract attention at all. It is just a matter of dosage that a professional examiner must take into account, and the preferred dosage of the Attention-Attracting-Baits should follow the Goldilocks Principle (Krapohl \& Shaw, 2015, p. 68; Ginton, 2019, p. 190).

The Goldilocks principle. It is derived from a children's story, "The Three Bears", in which a little girl named Goldilocks finds a house owned by three bears. Each bear has its own preference for food, beds, etc. After testing each of the three items, Goldilocks determines that one of them is always too much in one extreme, one is too much in the opposite extreme, and one is "just right". 
Whatever the polygraph case is, this principle stays the same, but the actual values of the "just right" level of the attention-attracting-baits must be changed to fit the individual subject and the specific circumstances.

Not adjusting the size or the degree of the baits to the case means discarding the real meaning of the Goldilocks Principle.

\section{The wise meaning of the Goldilocks Principle for CQT:}

- "Too strong" or "Too weak" baits are not fixed objective values, but rather case-depended matters, and so is the "Just Right".

- The examiner should adjust the size or the degree of the baits to the case.

- The difference between typical and great examiners lies in their capability to master this delicate matter.

- The RIG strength for a deceptive examinee is high, and therefore his attention is stuck in the relevant issue; however, under some circumstances, the RIG strength of truthful examinee is also very strong, and therefore he is prone to produce FP error unless the examiner will recognize the danger and adjust the pretest to increase the attractiveness of the Bait. Examples of such cases are alleged victim or a high-profile suspicion; if the examiner would not act to increase the weight of the bait, it is reasonable to expect a higher risk for FP. This is the meaning of applying Adaptive Polygraphy. "One size does not fit all" a good examiner should identify the difference between subjects and circumstances and adjust the test accordingly.

- "One size fits all, or else we lose standardization" is the motto of the Evidence-Based devotees in our profession, who, in the name of science, worship zealously the strict standardization that prevents chaos but also adversely affects flexibility and creativity. That means that one should not play with the amount or level of the Attention-Attracting-Baits from case to case, from one examinee to another.

- Conversely, in line with the RIG strength rationale, it is recommended to keep some flexibility and invest in deepening our understanding of the CQT by asking "WHY", developing new hypotheses, try them, and put them into objective tests.

- It should be stressed once more that the Deception Factor is not the only factor affecting the strength of the RIG. There are a variety of personal and circumstantial factors that also affect it, as shown in illustration 2. 


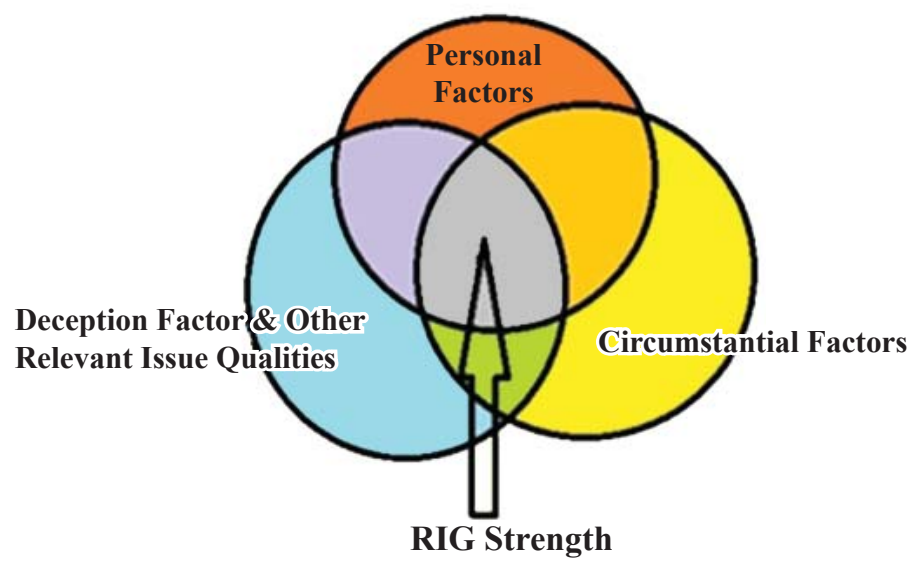

$\mathbf{R I G}=$ The competing force arising from an aggregation of qualities that the relevant issue possesses interacting with circumstantial and personal factors to capture and $b$. the examinee's attention.

Fig. 2. Factors affecting the RIG strength

All these factors are irrelevant for identifying deception or truthfulness, but they affect the RIG strength and, by that, influence the attention shifting processes between the relevant and the comparison spheres. Adaptive Polygraphy should take this into account and adjust the size of the baits set for attracting and shifting the attention away from the relevant issue towards the comparison sphere.

We should be aware of the existence of such factors in each case, and when we encountered a heavily loaded factor in a particular case, we must not ignore it in the name of objectivity and standardization. Instead, we should relate to it and adjust the pretest interview to suit that specific situation.

In particular, we should maneuver the level or the size of the bait that we are presenting in our effort to divert the examinee's attention from the relevant to the comparison sphere. That is to say, that the examiner should play with the amount of emphasis we/she put on the Comparison vs. the Relevant questions to balance the assumed effect of the identified extra factor on the RIG strength. In fact, this is the meaning of how to use the Goldilocks principle wisely in presenting the "Just Right" bait for optimizing the CQT outcomes. 
This might be a seed for developing in the polygraph profession, a scientific-based approach that does not refer to all sorts of variability as something to ignore or "fixed" statistically as if it were noise. Instead, variability should be recognized as a phenomenon that has to be treated with what I have termed "ADAPTIVE POLYGRAPHY," in which the polygraph testing procedures and dynamic will not be "one size fits all" but "Different Things to Different People and Different Circumstances”.

\section{Examples of Factors other than Lying Vs. Telling the Truth that might affect the RIG strength}

Some factors seem more likely to affect the RIG, while others seem unlikely to do so. Nevertheless, the idea of scientific-based Adaptive Polygraphy means to go beyond intuition into research that looks for evidence on both sides, the one supporting the existence of a phenomenon and the counter side that supports the lack of the phenomenon. Thus, if it seems, for instance, that age is not likely to affect the RIG, we still have to check it in order to rely on that in applying Adaptive Polygraphy.

\section{- Issue's Factors}

- Severity in terms of formal consequences (e.g., the expected punishment).

- Objective Emotional loads (e.g., minor sexual offense vs. minor theft).

- Personal Factors

- Age.

- Level of education.

- Previous criminal experience.

- Previous polygraph experience.

- Personality type or traits (e.g., obsessive vs. scatterbrained).

- Working status (e.g., manager vs. low-level worker).

- Social status (e.g., a teacher vs. a mechanic; celebrity vs. "no-body").

- Socioeconomic level.

- Circumstantial Factors

- Strength of Existing evidence.

- Depth and length of prior interrogation. 
- Public profile of the case (e.g., no one heard about vs. daily headlines).

- Same or different gender of the examiner.

- Ethnic issues.

\section{- Concrete examples}

- Alleged victim case.

- Witness to a traumatic event.

- Recidivist criminal.

- High profile case.

- Reexamination.

- ADD/ADHD -Attention Deficit (Hyperactive) Disorder

- OCD subjects.

- Serial offense.

- A criminal turns the state's evidence.

- Retracted admission.

- Suspecting false admission.

As mention before, a primary means in applying Adaptive Polygraphy is adjusting the baits for diverting the attention from the relevant to the comparison spheres. That should be done based on research rather than mere intuition; however, as long as no hard data available, it is suggested that instead of doing it intuitively, before starting the test, to screen the case along the categories mentioned above and estimate the expected impacts they would have on the RIG strength of the examinee at four levels (Low, Medium, High, Overwhelming). That should adjust the level by which you are to emphasize the comparison vs. the relevant issues and questions.

Of course, to do it, the examiner must be trained to identify the variance between subjects and circumstances and adapt the baits' strength to suit the appropriate balance between the relevant and the comparison questions in each particular test. It used to be part of the training but unfortunately, not anymore. It is about time to go back to this vital practice.

In most cases, we can only rely on our judgment of how particular facts affect the RIG strength; however, increased awareness of this notion and investment of research efforts in the forthcoming years might bring about research-supported information that will direct us in this regard. 
The adaptive Polygraphy should play a role in dealing with Idiosyncratic and "Paradoxical" Reactions such as Blood volume drop; Increased Pulse rate; Increased Pulse amplitude; Change in extra systolic beat rates, Hyperventilation; Irregular breathing; Fast breathing, and more so.

Research has shown that the response analysis will be better off without considering these reactions as valid responses for our purpose. Nevertheless, experience polygraph examiners have encountered cases in which these responses were significant. Research that will focus on these outliers might improve our understanding of the matter and our success rate by using adaptive analysis.

Along with the understanding that polygraph examiners should not function outside of any standardization or without having any scientifically proven support to their technique, we should beware not to narrow our steps and minds beyond the minimum necessary to avoid chaos.

Examiners should be able to practice their work with enough freedom to enable flexibility needed for adjusting the test to the specific examinee and circumstances, and during the years to achieve research support to the differential treating of the individual case. Interestingly enough, let me remind you that even when it comes to test data analysis (TDA), it is well documented that the rate of success achieved by the original examiners is higher than the one achieved by more objective analysis made by others (Raskin and Honts. 2002).

This is not the place to elaborate about the polygraph in court versus investigation. However, it is clear to the author that one of the enemies of the adaptive polygraph is the drive to bring the polygraph as evidence to the court. The constant squinting towards the court of law should be restrained. The proper place for the polygraph is the investigation dynamic and not the judicial arena. We as polygraph examiners are looking for the truth, not for justice, whereas the justice system is not bound to the truth alone but other values as well (see, for instance, the "Fruit of the poisonous tree' dilemma). As polygraph examiners, we cannot treat every examinee equally; we should give him an equal chance to let his version show up. For this to happen, we have to treat anyone in a tailor-made approach which by definition is not exactly the same in each case. On our way to reveal the truth keeping equal treatment to each case is wrong, whereas it is the right way to go with the judicial philosophy of the western culture. Hence, the bottom line is that the efforts to qualify for evidence in court are damaging to maximizing the polygraph potential. 


\section{How to avoid stepping on a slippery slope to Chaos?}

Intuitively, Adaptive Polygraphy seems to contain the danger of stepping on a wet slippery slope that might bring us back to the chaos that characterized the situation a few decades ago. Any examiner can do anything, and as long as he measures physiological reactions to questions or to other kinds of stimuli related to an investigated issue, he may claim to practice psychophysiological detection of deception. That is, of course, not what this concept means.

The "Adaptive Polygraphy" should strive to be a scientific-based approach that allows flexibility within the limits of scientific knowledge and/or scientific thinking and reasoning. It is an approach that looks at the variance among examinees and circumstances not as noise to ignore but as a source of information and insights that looking only under the street lamp with rigid or semi-rigid boundaries would not get us there.

How to control the danger of a wet slippery downwards embedded in the adaptive Polygraphy? This is not an easy mission, and it takes time to achieve. Fortunately, the danger is not an immediate one because, after a decade or two in which polygraph examiners were educated and pushed to adopt best practice methods in a variety of polygraph issues and got used to working within those frameworks, they are not eager to say the least, to leave their comfort zone and search for 'professional adventures. That gives us time to introduce the recommended change of Adaptive Polygraphy at a slow pace allowing for in-depth processes to take place.

The first in-depth change should happen in training polygraph examiners. It starts from the student admission. As it is currently practiced in most schools, people are admitted based on minimum qualifications: a Bachelor's degree, no criminal record, and being able to pay for a ten-week course. No one really cares about their cognitive, temperamental, and some other personality features, although it is recognized that those matters are crucial for being a good examiner. Unfortunately, some serious professionals think that having a good scientific test means to erase any humane touch that, by its very being humane, introduces variance to the test, which they see as a noise. If this is how they think about what a good test means, then no wonder we can and even should treat the candidates' personal suitability to become examiners as something to ignore on our way to becoming a scientific-based profession. There is no genuine evaluation and going-on process of selection during the course, and very few students who entered the first day do not get a school diploma from a qualified polygraph examiner when the course is over. Yes, 
they can spell out the basic history, legal status, and a few polygraph test formats with rigorous scoring methods. However, they hardly encountered any real dilemma or faced living polygraph cases that introduce the range of real-life diversity in subjects and circumstances. They are trained to believe that "one size fits all", or at most that there are a couple of models they have to choose between them and follow them to the dot. Also, the number of students per instructor is usually too high, and while it may suit the parts of the lecture, it is problematic when training exercises are concerned. That is not the case where training is more than a factory that produces examiners for profit. There are such places, but there are too many schools or polygraph training programs worldwide that do very poor work. Efforts to impose standards on schools have dealt with outside matters, i.e., the envelope of our profession, and failed to deal with the essence of the problem, which is a poor in-depth understanding of Polygraphy.

All of these should be changed, although, given the existing business factors in the equation, it will take lots of effort and patience. Nevertheless, we should start pushing in this direction, going through a mode of keeping humane touch, encouraging self-thinking, and deepening the understanding of the issue instead of obeying strict rules to their dots.

Quality control is a second important element in controlling the danger of the Adaptive Polygraph being a slippery slope towards chaos. Open-minded quality control of knowledgeable and experienced professionals should prevent messy examinations but enable caution variations that the examiner can explain its logic. Quality control is a place that can integrate new experiences and aggregated knowledge into a corpus that is more than anecdotal events. But that, of course, needs the right attitude, which is led by the Adaptive Polygraph approach.

\section{A closing remark}

One more word on the future of our profession - The psychophysiological Detection of Deception.

It is my belief that whether or not we turn to Adaptive Polygraphy in the next few years using our current psychophysiological measurements, the accelerating progress in brain research will bring us eventually to the Adaptive Polygraphy paradigm one way or another. The scientific detection of deception ought to become more versatile in order to improve its performance which means applying "Different Things to Different People and Different Situations". 


\section{References}

American Polygraph Association, (2011), Meta-Analytic Survey of Criterion Accuracy of validated Polygraph Techniques, Polygraph, 40 (4), 194-305.

Backster C. (1963), The Backster chart reliability rating method, Law and Order, 1, 63-64.

Elaad E. (1999), The Control Question Technique: A search for improved decision rules, Polygraph, 28, 65-73.

Ginton A. (2009), Relevant Issue Gravity (RIG) Strength - A new concept in PDD that reframes the notion of Psychological Set and the role of attention in CQT polygraph examinations, Polygraph, 38(3), 204-217

Ginton A. (2013, Sept), Adaptive Polygraph. [Paper Presentation]. The annual meeting of the American Polygraph Association, Orlando, FL. USA.

Ginton A. (2019), Essentials of the Relevant Issue Gravity (RIG) Strength; A Theoretical Framework for Understanding the Comparison Question Test (CQT); A detailed outline version, European Polygraph, 13 (4), 181-201

Honts C.R. and Driscoll L.N. (1987), An evaluation of the reliability and validity of rank order and standard numerical scoring of polygraph charts, Polygraph,16 (4), 241-257.

Keeler L. (1933), Scientific methods of criminal detection with the polygraph, KansasBar association, 2, 22-31.

Krapohl D.J. (2005), Polygraph decision rules for evidentiary and paired-testing (Marin Protocol) applications, Polygraph 34(3), 184-194.

Krapohl D.J. and Shaw P.K. (2015), Fundamental of polygraph practice, Academic Press.

Matte, J.A., (1996), Forensic Psychophysiology Using the Polygraph, J.A.M. Publications. Williamsville, NY

National Research Council (2003), The Polygraph and Lie Detection. Washington, DC: The National Academies Press. https://doi.org/10.17226

Raskin D.C., Honts C.R. and Kircher J.C. (eds.), (2014), Credibility Assessment: Scientific Research and Applications, eds. Elsevier, New York.

Raskin D.C. and Honts C.R. (2002), The Comparison Question Test. in Kleiner M. (Ed.), Handbook of Polygraph Testing, Academic Press, New York. 
Reid J.E. (1947), A revised questioning technique in lie detection tests, Journal of Criminal Law, Criminology and Police Science, 37, 542-547. 